\title{
NOTE ON THE GENERALIZED CONFORMABLE DERIVATIVE
}

\author{
ALBERTO FLEITAS, JUAN E. NÁPOLES VALDÉS, JOSÉ M. RODRÍGUEZ, \\ AND JOSÉ MARÍA SIGARRETA-ALMIRA
}

\begin{abstract}
We introduce a definition of a generalized conformable derivative of order $\alpha>0$ (where this parameter does not need to be integer), with which we overcome some deficiencies of known local derivatives, conformable or not. This definition allows us to compute fractional derivatives of functions defined on any open set on the real line (and not just on the positive halfline). Moreover, we extend some classical results to the context of fractional derivatives. Also, we obtain results for the case $\alpha>1$.
\end{abstract}

\section{Preliminaries}

The origins of fractional calculus date back to Newton and Leibniz in the 17th century, and thus, this branch of mathematical analysis can be considered as old as classical calculus (see 26]). Fractional calculus extends derivation and integration to arbitrary non-integer orders, meaning by that, rational, real or even complex orders. The fact is that fractional calculus, at present, is successfully used to model a broad range of phenomena that occur in physics, economy and science. For example, it has been observed that the time or space evolution of many physical processes can be more precisely described when derivatives of fractional order are introduced (see, e.g., [7, 8, 9, 10, 13, 2, 22, ). In practice, and in many occasions, all that needs to be done is replacing the time derivative in a given evolution equation by a derivative of fractional order. And the most interesting part is that this is not the result of chance; on the contrary, there is a strong mathematical foundation for it, as several studies show (see [4, 25, 18, 16, 19, 22, 2, 23, 24]). For a complementary study on the recent developments in the field of fractional calculus as well as its applications see [11, 6, 14, 27.

One of the most surprising and appealing parts of the theory is that classical fractional derivatives (e.g., Caputo and Riemann-Liouville) are not collecting merely local information. By contrast, fractional operators keep track of the history of the process being studied; this feature allows modeling the non-local and distributed responses that commonly appear in natural and physical phenomena.

2020 Mathematics Subject Classification. Primary 26A33; Secondary 34K37.

Key words and phrases. Fractional derivatives; fractional calculus; applications.

The research of José M. Rodríguez and José M. Sigarreta is supported by a grant from Agencia Estatal de Investigación (PID2019-106433GB-I00 / AEI / 10.13039/501100011033), Spain. 
On the other hand, one has to recognize that these fractional derivatives $D^{\alpha}$ show some serious drawbacks; among them:

(1) Most of the fractional derivatives (except Caputo-type), do not satisfy $D^{\alpha}(1)=0$, if $\alpha$ is not a natural number.

(2) Neither of the fractional derivatives satisfies the classical product rule for two functions, $D^{\alpha}(f g)=g D^{\alpha}(f)+f D^{\alpha}(g)$.

(3) Neither of the fractional derivatives satisfies the classical quotient rule for two functions, $D^{\alpha}\left(\frac{f}{g}\right)=\frac{g D^{\alpha}(f)-f D^{\alpha}(g)}{g^{2}}$, with $g \neq 0$.

(4) Neither of the fractional derivatives satisfies the chain rule for composite functions, $D^{\alpha}(f \circ g)(t)=D^{\alpha}(f(g)) D^{\alpha} g(t)$.

(5) Fractional derivatives do not have a corresponding "calculus".

(6) Neither of the fractional derivatives satisfies the indices rule, $D^{\alpha} D^{\beta}(f)=$ $D^{\alpha+\beta}(f)$.

A few years ago, some definitions of local fractional derivatives (firstly conformable, but also non-conformable more recently) were introduced. For this purpose, a certain incremental quotient was considered. In this way, a new direction in fractional calculus was opened.

The suitability of the adjective conformable in this context is questionable. Conformable fractional derivatives were initially defined so that $D^{\alpha} f(t) \rightarrow f^{\prime}(t)$ when $\alpha \rightarrow 1$; i.e., when $\alpha \rightarrow 1, D^{\alpha} f(t)$ preserves the angle of the tangent line to the curve, while in the non-conformable case this angle is not conserved.

Both in the conformable and in the non-conformable case, these local derivatives have been defined in a very limited range; basically, for $0<\alpha<1$. Actually, the attempts to extend them for orders beyond that interval are, certainly, very scarce.

In this paper, we present a definition of a generalized conformable derivative of order $\alpha>0$ (where $\alpha$ is allowed to take either integer or non-integer values), with which the aforementioned deficiencies are avoided and that fills the gap of derivatives of higher order, so far non-existent. This definition allows us to compute fractional derivatives of functions defined on any open set on the real line (and not just on the positive half-line). Moreover, we extend some classical results to the context of fractional derivatives. Also, we obtain results for the case $\alpha>1$.

This paper relies on the introduction and use of a local fractional derivative $G_{T}^{\alpha}$, depending on a general kernel function $T(t, \alpha)$, which includes at once several fractional derivatives introduced and studied earlier in [22, 17, 3]. More specifically:

(1) If $\alpha \in(0,1]$ and $T(t, \alpha)=t^{1-\alpha}$, then the conformable fractional derivative defined in [22] is obtained.

(2) If $\alpha \in(0,1]$ and $T(t, \alpha)=k(t)^{1-\alpha}$, then the general conformable fractional derivative defined in $[3$ is obtained.

(3) If $\alpha \in(0,1]$ and $T(t, \alpha)=e^{t^{-\alpha}}$, then the non-conformable fractional derivative defined in [17] is obtained. 


\section{MAIN RESUltS}

In this section, a new definition of a local generalized fractional derivative of a function at a point $t$ is introduced, and some results similar to those of classical calculus are obtained.

Given $s \in \mathbb{R}$, we denote by $\lceil s\rceil$ the upper integer part of $s$, i.e., the smallest integer greater than or equal to $s$.

Definition 2.1. Given an interval $I \subseteq \mathbb{R}, f: I \rightarrow \mathbb{R}, \alpha \in \mathbb{R}^{+}$and a positive continuous function $T(t, \alpha)$ on $I$, the derivative $G_{T}^{\alpha} f$ of $f$ of order $\alpha$ at the point $t \in I$ is defined by

$$
G_{T}^{\alpha} f(t)=\lim _{h \rightarrow 0} \frac{1}{h^{\lceil\alpha\rceil}} \sum_{k=0}^{\lceil\alpha\rceil}(-1)^{k}\left(\begin{array}{c}
\lceil\alpha\rceil \\
k
\end{array}\right) f(t-k h T(t, \alpha)) .
$$

If $a=\min \{t \in I\}$ (respectively, $b=\max \{t \in I\}$ ), then $G_{T}^{\alpha} f(a)$ (respectively, $\left.G_{T}^{\alpha} f(b)\right)$ is defined with $h \rightarrow 0^{-}$(respectively, $h \rightarrow 0^{+}$) instead of $h \rightarrow 0$ in the limit.

If we choose the function $T(t, \alpha)=t^{\lceil\alpha\rceil-\alpha}$, then we obtain the following particular case of $G_{T}^{\alpha}$. Note that $T(t, \alpha)=t^{\lceil\alpha\rceil-\alpha}=1$ for every $\alpha \in \mathbb{N}$.

Definition 2.2. Let $I \subseteq(0, \infty)$ be an interval, $f: I \rightarrow \mathbb{R}$ and $\alpha \in \mathbb{R}^{+}$. The conformable derivative $G^{\alpha} f$ of $f$ of order $\alpha$ at the point $t \in I$ is defined by

$$
G^{\alpha} f(t)=\lim _{h \rightarrow 0} \frac{1}{h^{\lceil\alpha\rceil}} \sum_{k=0}^{\lceil\alpha\rceil}(-1)^{k}\left(\begin{array}{c}
\lceil\alpha\rceil \\
k
\end{array}\right) f\left(t-k h t^{\lceil\alpha\rceil-\alpha}\right) .
$$

We know from the classical calculus that if $f$ is a function defined in a neighborhood of the point $t$, and there exists $D^{n} f(t)$, then

$$
D^{n} f(t)=\lim _{h \rightarrow 0} \frac{1}{h^{n}} \sum_{k=0}^{n}(-1)^{k}\left(\begin{array}{l}
n \\
k
\end{array}\right) f(t-k h) .
$$

Therefore, if $\alpha=n \in \mathbb{N}$ and $f$ is smooth enough, then Definition 2.2 coincides with the classical definition of the $n$-th derivative.

In [22] a conformable derivative is defined in the following way. Given $f$ : $(0, \infty) \rightarrow \mathbb{R}$ and $\alpha \in(0,1]$, the derivative of $f$ of order $\alpha$ at the point $t$ is defined by

$$
T_{\alpha} f(t)=\lim _{h \rightarrow 0} \frac{f(t)-f\left(t-h t^{1-\alpha}\right)}{h} .
$$

It is clear then that $T_{\alpha}$ is a particular case of $G^{\alpha}$ when $\alpha \in(0,1]$ and $T(t, \alpha)=t^{1-\alpha}$. See [1, 20, 21] for more information on $T_{\alpha}$.

In [22] the following local fractional derivative is defined.

Definition 2.3. Given $f:(0, \infty) \rightarrow \mathbb{R}$, the derivative $T_{\alpha} f$ of $f$ of order $\alpha \in \mathbb{R}^{+}$is defined by

$$
T_{\alpha} f(t)=\lim _{h \rightarrow 0} \frac{f^{(\lceil\alpha\rceil-1)}(t)-f^{(\lceil\alpha\rceil-1)}\left(t-h t^{\lceil\alpha\rceil-\alpha}\right)}{h} .
$$


We can establish the following differences between our definition and the generalized derivative $T_{\alpha}$ :

(1) Definition 2.1 permits direct inferences for the fractional derivative of any positive order, whereas in Definition 2.3, the fractional derivative can be applied only to the last derivative.

(2) Definition 2.3 can be applied only to functions defined on a subset of $\mathbb{R}^{+}$, and the generality of Definition 2.1 allows us to consider fractional derivatives of functions defined on $\mathbb{R}$.

We start by proving the following result.

Theorem 2.4. Let $I \subseteq \mathbb{R}$ be an interval, $f: I \rightarrow \mathbb{R}$ and $\alpha \in \mathbb{R}^{+}$.

(1) If there exists $D^{\lceil\alpha\rceil} f$ at the point $t \in I$, then $f$ is $G_{T}^{\alpha}$-differentiable at $t$ and $G_{T}^{\alpha} f(t)=T(t, \alpha)^{\lceil\alpha\rceil} D^{\lceil\alpha\rceil} f(t)$.

(2) If $\alpha \in(0,1]$, then $f$ is $G_{T}^{\alpha}$-differentiable at $t \in I$ if and only if $f$ is differentiable at $t$; in this case, we have $G_{T}^{\alpha} f(t)=T(t, \alpha) f^{\prime}(t)$.

Proof. Assume first that there exists $D^{\lceil\alpha\rceil} f$ at the point $t$. If we take $q=h T(t, \alpha)$ in the definition of $G_{T}^{\alpha} f$, then we obtain

$$
\begin{aligned}
G_{T}^{\alpha} f(t) & =T(t, \alpha)^{\lceil\alpha\rceil} \lim _{q \rightarrow 0} \frac{1}{q^{\lceil\alpha\rceil}} \sum_{k=0}^{\lceil\alpha\rceil}(-1)^{k}\left(\begin{array}{c}
\lceil\alpha\rceil \\
k
\end{array}\right) f(t-k q) \\
& =T(t, \alpha)^{\lceil\alpha\rceil} D^{\lceil\alpha\rceil} f(t) .
\end{aligned}
$$

Assume now that $\alpha \in(0,1]$. Thus, $\lceil\alpha\rceil=1$ and the previous argument gives

$$
G_{T}^{\alpha} f(t)=T(t, \alpha) \lim _{q \rightarrow 0} \frac{f(t)-f(t-q)}{q}=T(t, \alpha) f^{\prime}(t),
$$

and there exists $G_{T}^{\alpha} f(t)$ if and only if there exists $f^{\prime}(t)$.

The following result contains some basic properties of the derivative $G_{T}^{\alpha}$.

Theorem 2.5. Let $I \subseteq \mathbb{R}$ be an interval, $f, g: I \rightarrow \mathbb{R}$ and $\alpha \in \mathbb{R}^{+}$. Assume that $f, g$ are $G_{T}^{\alpha}$-differentiable functions at $t \in I$. Then the following statements hold:

(1) $a f+b g$ is $G_{T}^{\alpha}$-differentiable at $t$ for every $a, b \in \mathbb{R}$, and $G_{T}^{\alpha}(a f+b g)(t)=$ $a G_{T}^{\alpha} f(t)+b G_{T}^{\alpha} g(t)$.

(2) If $\alpha \in(0,1]$, then $f g$ is $G_{T}^{\alpha}$-differentiable at $t$ and $G_{T}^{\alpha}(f g)(t)=f(t) G_{T}^{\alpha} g(t)+$ $g(t) G_{T}^{\alpha} f(t)$.

(3) If $\alpha \in(0,1]$ and $g(t) \neq 0$, then $f / g$ is $G_{T}^{\alpha}$-differentiable at $t$ and $G_{T}^{\alpha}\left(\frac{f}{g}\right)(t)=$ $\frac{g(t) G_{T}^{\alpha} f(t)-f(t) G_{T}^{\alpha} g(t)}{g(t)^{2}}$.

(4) $G_{T}^{\alpha}(\lambda)=0$, for every $\lambda \in \mathbb{R}$.

(5) $G_{T}^{\alpha}\left(t^{p}\right)=\frac{\Gamma(p+1)}{\Gamma(p-\lceil\alpha\rceil+1)} t^{p-\lceil\alpha\rceil} T(t, \alpha)^{\lceil\alpha\rceil}$, for every $p \in \mathbb{R} \backslash \mathbb{Z}^{-}$.

(6) $G_{T}^{\alpha}\left(t^{-n}\right)=(-1)^{\lceil\alpha\rceil} \frac{\Gamma(n+\lceil\alpha\rceil)}{\Gamma(n)} t^{-n-\lceil\alpha\rceil} T(t, \alpha)^{\lceil\alpha\rceil}$, for every $n \in \mathbb{Z}^{+}$. 
Proof. Items (1) and (4) follow from the definition of $G_{T}^{\alpha}$.

If $\alpha \in(0,1]$, then Theorem 2.4 gives that $f, g$ are differentiable functions at $t$, and $G_{T}^{\alpha} h(t)=T(t, \alpha) h^{\prime}(t)$ for any differentiable function $h$. These facts imply items $(2)$ and $(3)$.

Finally, since $f(t)=t^{p}$ is a $C^{\infty}$ function on $(0, \infty)$, Theorem 2.4 gives $G_{T}^{\alpha}\left(t^{p}\right)=$ $T(t, \alpha)^{\lceil\alpha\rceil} D^{\lceil\alpha\rceil}\left(t^{p}\right)$.

Although Theorem 2.4 provides a way to obtain $G^{\alpha} f$, unfortunately the index law for iterated derivatives is not fulfilled. In this direction we propose a general rule in order to compute iterated conformable derivatives.

Theorem 2.6. Let $I \subseteq(0, \infty)$ be an interval, $f: I \rightarrow \mathbb{R}$ and $\alpha_{1}, \alpha_{2}, \ldots, \alpha_{n} \in(0,1]$. If there exists $D^{n} f(t)$ for some $t \in I$, then

$$
G^{\alpha_{n}} \cdots G^{\alpha_{2}} G^{\alpha_{1}} f(t)=t^{-\alpha_{1}-\alpha_{2}-\cdots-\alpha_{n}} \sum_{k=1}^{n} b_{n, k} t^{k} f^{(k)}(t)
$$

with

$$
\begin{array}{ll}
b_{n, n}=1, b_{n, 0}=0, & \text { for } n \geq 1, \\
b_{n, k}=\left(k-\alpha_{1}-\cdots-\alpha_{n-1}\right) b_{n-1, k}+b_{n-1, k-1}, & \text { for } k<n, \\
b_{n, 1}=\prod_{j=1}^{n-1}\left(1-\alpha_{1}-\cdots-\alpha_{j-1}\right), & \text { for } n>1 .
\end{array}
$$

Proof. Since $T(t, \alpha)=t^{1-\alpha}$ for $\alpha \in(0,1]$, Theorem 2.4 gives the result if $n=1$.

Assume that the result holds for $n-1$ and let us prove that it holds for $n$. Theorem 2.4 gives

$$
\begin{aligned}
G^{\alpha_{n}} \cdots G^{\alpha_{2}} G^{\alpha_{1}} f(t)= & G^{\alpha_{n}}\left(\sum_{k=1}^{n-1} b_{n-1, k} t^{k-\alpha_{1}-\cdots-\alpha_{n-1}} f^{(k)}(t)\right) \\
= & \sum_{k=1}^{n-1} b_{n-1, k}\left(k-\alpha_{1}-\cdots-\alpha_{n-1}\right) t^{k-\alpha_{1}-\cdots-\alpha_{n-1}-1} t^{1-\alpha_{n}} f^{(k)}(t) \\
& +\sum_{k=1}^{n-1} b_{n-1, k} t^{k-\alpha_{1}-\cdots-\alpha_{n-1}} t^{1-\alpha_{n}} f^{(k+1)}(t) \\
= & \sum_{k=1}^{n-1} b_{n-1, k}\left(k-\alpha_{1}-\cdots-\alpha_{n-1}\right) t^{k-\alpha_{1}-\cdots-\alpha_{n}} f^{(k)}(t) \\
& +\sum_{k=2}^{n} b_{n-1, k-1} t^{k-\alpha_{1}-\cdots-\alpha_{n}} f^{(k)}(t),
\end{aligned}
$$


and this gives $b_{n, k}=\left(k-\alpha_{1}-\cdots-\alpha_{n-1}\right) b_{n-1, k}+b_{n-1, k-1}$ for $k<n$ (since $\left.b_{n, 0}=0\right)$ and $b_{n, n}=b_{n-1, n-1}=1$. Also,

$$
\begin{aligned}
b_{n, 1} & =\left(1-\alpha_{1}-\cdots-\alpha_{n-1}\right) b_{n-1,1} \\
& =\left(1-\alpha_{1}-\cdots-\alpha_{n-1}\right) \prod_{j=1}^{n-2}\left(1-\alpha_{1}-\cdots-\alpha_{j-1}\right) \\
& =\prod_{j=1}^{n-1}\left(1-\alpha_{1}-\cdots-\alpha_{j-1}\right)
\end{aligned}
$$

for $n>1$, and this finishes the proof.

In [12], the previous result is generalized as follows.

Theorem 2.7. Let $I \subseteq \mathbb{R}$ be an interval, $t \in I, f: I \rightarrow \mathbb{R}, n \geq 2$ and $\alpha_{1}, \alpha_{2}, \ldots, \alpha_{n} \in(0,1]$. Assume that $T^{(n-k)}\left(t, \alpha_{k}\right)$ exists for $1 \leq k \leq n-1$. Let us define $\bar{T}_{k}=\left(T\left(t, \alpha_{k}\right), T^{\prime}\left(t, \alpha_{k}\right), T^{\prime \prime}\left(t, \alpha_{k}\right), \ldots, T^{(n-k)}\left(t, \alpha_{k}\right)\right)$ if $1 \leq k<n$, and $\bar{T}_{n}=T\left(t, \alpha_{n}\right)$. Then $G_{T}^{\alpha_{n}} G_{T}^{\alpha_{n-1}} \cdots G_{T}^{\alpha_{1}} f(t)$ exists if and only if $f^{(n)}(t)$ exists, and we have in this case

$$
G_{T}^{\alpha_{n}} G_{T}^{\alpha_{n-1}} \cdots G_{T}^{\alpha_{1}} f(t)=Q_{n}(t) f^{(n)}(t)+P_{n}(t),
$$

where $P_{n}=p_{n}\left(\bar{T}_{1}, \ldots, \bar{T}_{n}, f^{\prime}, \ldots, f^{(n-1)}\right)$ and $p_{n}$ is a polynomial on $\left(n^{2}+3 n-2\right) / 2$ variables which is homogeneous of degree $n+1$ and

$$
Q_{n}(t)=T\left(t, \alpha_{n}\right) T\left(t, \alpha_{n-1}\right) \cdots T\left(t, \alpha_{2}\right) T\left(t, \alpha_{1}\right) .
$$

Theorem 2.4 has the following consequences.

Proposition 2.8. Let $I \subseteq \mathbb{R}$ be an interval, $f: I \rightarrow \mathbb{R}$ and $\alpha \in \mathbb{R}^{+}$. If there exists $D^{\lceil\alpha\rceil} f$ on $I$ and $G_{T}^{\alpha} f=0$ on $I$, then $f$ is a polynomial of degree at most $\lceil\alpha\rceil-1$ on $I$.

Proposition 2.9. Let $f$ be a function with a local extreme at $t_{0}$. If $f$ is $G_{T}^{\alpha-}$ differentiable on $t_{0}$ for some $\alpha \in(0,1]$, then $G_{T}^{\alpha}\left(t_{0}\right)=0$.

Note that if $T(t, n)=1$ when $n \in \mathbb{N}$ (in particular, if $T(t, \alpha)=t^{\lceil\alpha\rceil-\alpha}$ ) and there exists $D^{n} f(t)$, then Theorem 2.4 gives $G_{T}^{n} f(t)=D^{n} f(t)$.

An argument similar to the one in the proof of Theorem 2.5 provides the chain rule for this fractional derivative.

Theorem 2.10. Let $\alpha \in(0,1], g$ a $G_{T}^{\alpha}$-differentiable function at $t$ and $f$ a differentiable function at $g(t)$. Then $f \circ g$ is $G_{T}^{\alpha}$-differentiable at $t$, and $G_{T}^{\alpha}(f \circ g)(t)=$ $f^{\prime}(g(t)) G_{T}^{\alpha} g(t)$.

Following [22], if $f:[0, \infty) \rightarrow \mathbb{R}$, then we define $G^{\alpha} f(0)=\lim _{t \rightarrow 0^{+}} G^{\alpha} f(t)$ when this limit exists (in this case it is not possible to apply Definition 2.2 since $T(t, \alpha)=t^{\lceil\alpha\rceil-\alpha}$ and $\left.T(0, \alpha)=0\right)$. Note that the statement in Theorem 2.4 does not hold in general for $G^{\alpha} f(0)$. 
Proposition 2.11. For each positive integer $n$ and $\alpha \in(n-1, n)$ such that $n(n-$ $\alpha) \notin \mathbb{Z}$, there are functions $f:[0, \infty) \rightarrow \mathbb{R}$ such that $f$ is $G^{\alpha}$-differentiable on $[0, \infty)$ and $D^{\lceil n(1-(n-\alpha))\rceil} f(0)$ does not exist.

Proof. Recall that $G^{\alpha}=G_{T}^{\alpha}$ with $T(t, \alpha)=t^{n-\alpha}$. Theorem 2.5 gives $G^{\alpha}\left(t^{p}\right)=$ $\frac{\Gamma(p+1)}{\Gamma(p+1-n)} t^{p-n}\left(t^{n-\alpha}\right)^{n}$, for every $p \in \mathbb{R} \backslash \mathbb{Z}^{-}$and $t>0$. If we choose $p=n(1-(n-\alpha))$, then we have

$$
G^{\alpha}\left(t^{p}\right)=\frac{\Gamma(p+1)}{\Gamma(p+1-n)} t^{p-n}\left(t^{n-\alpha}\right)^{n}=\frac{\Gamma(p+1)}{\Gamma(p+1-n)}
$$

for every $t>0$ and so for every $t \geq 0$.

Since $p=n(1-(n-\alpha)) \notin \mathbb{Z}$, we have that $\left(D^{\lceil p\rceil} t^{p}\right)(0)=\left(D^{\lceil n(1-(n-\alpha))\rceil} t^{p}\right)(0)$ does not exist, and this finishes the proof.

If we take $\alpha=n-1 / 2$ in Proposition 2.11, then we obtain the following result.

Corollary 2.12. For each odd integer $n$ greater than 1 , there are functions $f$ : $[0, \infty) \rightarrow \mathbb{R}$ such that $f$ is $G^{n-1 / 2}$-differentiable on $[0, \infty)$ and $D^{(n+1) / 2} f(0)$ does not exist.

As an application of fractional calculus, the following result provides a new proof of some known combinatorial identities.

Proposition 2.13. For each pair of positive integers $r<n$ we have

$$
\sum_{k=0}^{n}\left(\begin{array}{l}
n \\
k
\end{array}\right)(-1)^{k} k^{r}=0, \quad \sum_{k=0}^{n}\left(\begin{array}{l}
n \\
k
\end{array}\right)(-1)^{k} k^{n}=(-1)^{n} n ! .
$$

Proof. For each fixed $n$, let us choose $\alpha \in(n-1, n) \backslash \mathbb{Q}$; thus, $p=n(1-(n-\alpha)) \notin \mathbb{Z}$. Let $g:[0, \infty) \rightarrow \mathbb{R}$ be the function $g(t)=t^{p}$. The argument in the proof of Proposition 2.11 gives

$$
G^{\alpha} g(t)=\frac{\Gamma(p+1)}{\Gamma(p+1-n)} \neq 0
$$

for every $t \geq 0$, since $p \notin \mathbb{Z}$. We have

$$
\frac{\Gamma(p+1)}{\Gamma(p+1-n)}=G^{\alpha} g(1)=\lim _{h \rightarrow 0} h^{-n} \sum_{k=0}^{n}(-1)^{k}\left(\begin{array}{l}
n \\
k
\end{array}\right)(1-k h)^{p} .
$$

If

$$
w(h)=\sum_{k=0}^{n}\left(\begin{array}{l}
n \\
k
\end{array}\right)(-1)^{k}(1-k h)^{p},
$$

then

$$
D^{r} w(h)=c_{p, r} \sum_{k=0}^{n}\left(\begin{array}{l}
n \\
k
\end{array}\right)(-1)^{k+r}(1-k h)^{p-r} k^{r},
$$

with $r<n(r \in \mathbb{N})$, and

$$
c_{p, r}=p(p-1)(p-2) \cdots(p-(r-1))=\frac{\Gamma(p+1)}{\Gamma(p+1-r)} .
$$


Note that $c_{p, r} \neq 0$, since $p \notin \mathbb{Z}$. Hence, by applying Bernoulli-L'Hôpital's rule $n$ times, we obtain

$$
\begin{aligned}
c_{p, n} & =G^{\alpha} g(1)=\lim _{h \rightarrow 0} \frac{c_{p, r}(-1)^{r} \sum_{k=0}^{n}\left(\begin{array}{l}
n \\
k
\end{array}\right)(-1)^{k}(1-k h)^{p-r} k^{r}}{(n-r) ! n(n-1) \cdots(n+1-r)} \\
& =\lim _{h \rightarrow 0} \frac{c_{p, n}(-1)^{n} \sum_{k=0}^{n}\left(\begin{array}{l}
n \\
k
\end{array}\right)(-1)^{k}(1-k h)^{p-n} k^{n}}{n !} \\
& =c_{p, n}(-1)^{n} \frac{\sum_{k=0}^{n}\left(\begin{array}{c}
n \\
k
\end{array}\right)(-1)^{k} k^{n}}{n !} .
\end{aligned}
$$

Hence,

$$
\begin{aligned}
& \sum_{k=0}^{n}\left(\begin{array}{l}
n \\
k
\end{array}\right)(-1)^{k} k^{r}=\lim _{h \rightarrow 0} \sum_{k=0}^{n}\left(\begin{array}{l}
n \\
k
\end{array}\right)(-1)^{k}(1-k h)^{p-r} k^{r}=0, \\
& \sum_{k=0}^{n}\left(\begin{array}{l}
n \\
k
\end{array}\right)(-1)^{k} k^{n}=(-1)^{n} n !
\end{aligned}
$$

Note that there are important differences between the results obtained in the case of $\alpha \in(0,1]$, which are generalizations of classical theorems, and the (unexpected) results obtained for $\alpha \in(1,2]$ (see for example Theorem 2.14 and Proposition 2.16).

Theorem 2.14. Let $I \subseteq \mathbb{R}$ be an interval, $f: I \rightarrow \mathbb{R}$ and $\alpha, \beta \in \mathbb{R}^{+}$with $\lceil\alpha\rceil=$ $\lceil\beta\rceil$. Then $f$ is $G_{T}^{\alpha}$-differentiable at $t \in I$ if and only if it is $G_{T}^{\beta}$-differentiable at $t$, and furthermore,

$$
G_{T}^{\alpha} f(t)=\left(\frac{T(t, \alpha)}{T(t, \beta)}\right)^{\lceil\alpha\rceil} G_{T}^{\beta} f(t) .
$$

In particular, $f$ is $G_{T}^{\alpha}$-differentiable if and only if it is $G_{T}^{\lceil\alpha\rceil}$-differentiable.

Proof. Since $\lceil\alpha\rceil=\lceil\beta\rceil$, if we take $q=h T(t, \alpha)$ in the definition of $G_{T}^{\alpha} f$, then we obtain

$$
\begin{aligned}
G_{T}^{\alpha} f(t) & =T(t, \alpha)^{\lceil\alpha\rceil} \lim _{q \rightarrow 0} \frac{1}{q^{\lceil\alpha\rceil}} \sum_{k=0}^{\lceil\alpha\rceil}(-1)^{k}\left(\begin{array}{c}
\lceil\alpha\rceil \\
k
\end{array}\right) f(t-k q) \\
& =\left(\frac{T(t, \alpha)}{T(t, \beta)}\right)^{\lceil\alpha\rceil} T(t, \beta)^{\lceil\alpha\rceil} \lim _{q \rightarrow 0} \frac{1}{q^{\lceil\beta\rceil}} \sum_{k=0}^{\lceil\beta\rceil}(-1)^{k}\left(\begin{array}{c}
\lceil\beta\rceil \\
k
\end{array}\right) f(t-k q) \\
& =\left(\frac{T(t, \alpha)}{T(t, \beta)}\right)^{\lceil\alpha\rceil} G_{T}^{\beta} f(t),
\end{aligned}
$$

and there exists $G_{T}^{\alpha} f(t)$ if and only if there exists $G_{T}^{\beta} f(t)$.

Proposition 2.15. Given any $t_{0} \in \mathbb{R}$, there exists a function $g: \mathbb{R} \rightarrow \mathbb{R}$ which is $G_{T}^{\alpha}$-differentiable at $t_{0}$ for every $\alpha>1$, and which is not $G_{T}^{\alpha}$-differentiable at $t_{0}$ for $\alpha \in(0,1]$. 
Proof. Let us define a function $f: \mathbb{R} \rightarrow \mathbb{R}$ as follows: $f(t)=0$ if $t \in \mathbb{Q}$ and $f(t)=t$ otherwise.

Since $f$ is not differentiable at 0 , Theorem 2.4 gives that $f$ is not $G_{T}^{\alpha}$-differentiable at 0 for $\alpha \in(0,1]$.

Since $-h \in \mathbb{Q}$ if and only if $-k h \in \mathbb{Q}$ for every $k \in \mathbb{Z}^{+}$, we have for every $h \in \mathbb{R}$,

$$
\sum_{k=0}^{n}(-1)^{k}\left(\begin{array}{l}
n \\
k
\end{array}\right) f(-k h)=0
$$

for every $n \in \mathbb{N}$ with $n \geq 2$. Thus, we have

$$
\sum_{k=0}^{n}(-1)^{k}\left(\begin{array}{l}
n \\
k
\end{array}\right) f(-k h T(0, \alpha))=0
$$

for every $h \in \mathbb{R}$ and $n \in \mathbb{N}$ with $n \geq 2$. Hence, $f$ is $G_{T}^{n}$-differentiable at 0 for every $n \in \mathbb{N}$ with $n \geq 2$, and so Theorem 2.14 gives that $f$ is $G_{T}^{\alpha}$-differentiable at 0 for every $\alpha>1$.

Finally, given any $t_{0} \in \mathbb{R}$, the function $g(t)=f\left(t-t_{0}\right)$ is $G_{T}^{\alpha}$-differentiable at $t_{0}$ for every $\alpha>1$, and it is not $G_{T}^{\alpha}$-differentiable at $t_{0}$ for $\alpha \in(0,1]$.

Proposition 2.16. Given any $t_{0} \in \mathbb{R}$, there exists a $G_{T}^{\alpha}$-differentiable function $g: \mathbb{R} \rightarrow \mathbb{R}$ at $t_{0}$ for every $\alpha \in(1,2]$, and which is not continuous at $t_{0}$.

Proof. Consider the sequence of prime numbers $\left\{p_{k}\right\}_{k=1}^{\infty}$ greater than 2. Let us define a function $f: \mathbb{R} \rightarrow \mathbb{R}$ as follows:

$$
\begin{aligned}
f\left(-\frac{1}{2^{n} p_{k}}\right) & =\frac{p_{k}}{2^{n}}, \quad \text { for } k \geq 1, n \in \mathbb{Z}, \\
f(t) & =0, \quad \text { otherwise. }
\end{aligned}
$$

Since

$$
\lim _{k \rightarrow \infty} f\left(-\frac{1}{p_{k}}\right)=\lim _{k \rightarrow \infty} p_{k}=\infty
$$

$f$ is not continuous at 0 .

If $q=1 /\left(2^{n} p_{k}\right)$ for some $k \geq 1, n \in \mathbb{Z}$, then

$$
f(0)-2 f\left(-\frac{1}{2^{n} p_{k}}\right)+f\left(-\frac{1}{2^{n-1} p_{k}}\right)=-2 \frac{p_{k}}{2^{n}}+\frac{p_{k}}{2^{n-1}}=0,
$$

and so $f(0)-2 f(-q)+f(-2 q)=0$ for every $q \in \mathbb{R}$. Hence, for each $\alpha \in(1,2]$ and $h \in \mathbb{R}$, we have

$$
f(0)-2 f(-h T(0, \alpha))+f(-2 h T(0, \alpha))=0,
$$

and so $G_{T}^{\alpha} f(0)=0$ for every $\alpha \in(1,2]$.

Therefore, given any $t_{0}>0$, the function $g(t)=f\left(t-t_{0}\right)$ is $G_{T}^{\alpha}$-differentiable at $t_{0}$ for every $\alpha \in(1,2]$, and it is not continuous at $t_{0}$.

Theorem 2.17. Let $f:[a, b] \rightarrow \mathbb{R}$ be a continuous function such that $f(a)=f(b)$ and $f$ is $G_{T}^{\alpha}$-differentiable on $(a, b)$ for some $\alpha \in(0,1]$. Then there exists $c \in(a, b)$ such that $G_{T}^{\alpha} f(c)=0$. 
Proof. Since $f$ is continuous on $[a, b]$, and $f(a)=f(b)$, there is at least a point of local extreme $c \in(a, b)$. Since $f$ is $G_{T}^{\alpha}$-differentiable at $c$ with $\alpha \in(0,1]$, we have

$$
\begin{aligned}
G_{T}^{\alpha} f(c) & =G_{T}^{\alpha} f\left(c^{+}\right)=\lim _{h \rightarrow 0^{+}} \frac{f(c)-f(c-h T(c, \alpha))}{h} \\
& =G_{T}^{\alpha} f\left(c^{-}\right)=\lim _{h \rightarrow 0^{-}} \frac{f(c)-f(c-h T(c, \alpha))}{h} .
\end{aligned}
$$

Since $T(c, \alpha)>0$ and $c$ is a point of local extreme of $f$, we conclude that $G_{T}^{\alpha} f\left(c^{+}\right)$ and $G_{T}^{\alpha} f\left(c^{-}\right)$have opposite signs, and so Proposition 2.9 gives $G_{T}^{\alpha} f(c)=0$.

Theorem 2.18. Let $f:[a, b] \rightarrow \mathbb{R}$ be a continuous function, $G_{T}^{\alpha}$-differentiable on $(a, b)$ for some $\alpha \in(0,1]$. Then there exists $c \in(a, b)$ such that

$$
\frac{c^{1-\alpha}}{\alpha T(c, \alpha)} G_{T}^{\alpha} f(c)=\frac{f(b)-f(a)}{b^{\alpha}-a^{\alpha}} .
$$

Proof. Let us consider the function

$$
g(t)=f(t)-f(a)-\frac{f(b)-f(a)}{b^{\alpha}-a^{\alpha}}\left(t^{\alpha}-a^{\alpha}\right) .
$$

This function $g$ satisfies the hypotheses of Rolle's theorem (Theorem 2.17) and, therefore, there exists $c \in(a, b) \subset(0, \infty)$ such that $G_{T}^{\alpha} g(c)=0$. Then we have

$$
\begin{aligned}
G_{T}^{\alpha} f(t) & =G_{T}^{\alpha}\left(g(t)+f(a)+\frac{f(b)-f(a)}{b^{\alpha}-a^{\alpha}}\left(t^{\alpha}-a^{\alpha}\right)\right) \\
& =G_{T}^{\alpha} g(t)+\frac{f(b)-f(a)}{b^{\alpha}-a^{\alpha}} G_{T}^{\alpha}\left(t^{\alpha}\right), \\
G_{T}^{\alpha} f(c) & =\frac{f(b)-f(a)}{b^{\alpha}-a^{\alpha}} G_{T}^{\alpha}\left(t^{\alpha}\right)(c) .
\end{aligned}
$$

Since $\alpha \in(0,1]$, Theorem 2.4 gives $G_{T}^{\alpha}\left(t^{\alpha}\right)=T(t, \alpha) \alpha t^{\alpha-1}$, and we conclude that

$$
G_{T}^{\alpha} f(c)=T(c, \alpha) \alpha c^{\alpha-1} \frac{f(b)-f(a)}{b^{\alpha}-a^{\alpha}} .
$$

Theorem 2.19. Let $f:[a, b] \rightarrow \mathbb{R}$ be a continuous function, $G_{T}^{\alpha}$-differentiable on $(a, b)$ for some $\alpha \in(0,1]$. If $G_{T}^{\alpha} f(t)=0$ for all $t \in(a, b)$, then $f$ is constant on $[a, b]$.

Proof. Let us consider $s, t \in[a, b]$ with $s \neq t$. Without loss of generality we can assume that $s<t$. By Theorem 2.18, there exists $c \in(s, t) \subseteq(a, b) \subset(0, \infty)$ such that

$$
\frac{c^{1-\alpha}}{\alpha T(c, \alpha)} G_{T}^{\alpha} f(c)=\frac{f(t)-f(s)}{t^{\alpha}-s^{\alpha}} .
$$

Since $G_{T}^{\alpha} f(c)=0$, we conclude that $f(s)=f(t)$, and so $f$ is constant on $[a, b]$.

Corollary 2.20. Let $f$ be a continuous function on $[a, b]$ which is $G_{T}^{\alpha}$-differentiable on $(a, b)$ for some $\alpha \in(0,1]$. Then $G_{T}^{\alpha} f(t) \geq 0$ (respectively, $\leq 0$ ) if and only if $f$ is a non-decreasing (respectively, non-increasing) function. If $G_{T}^{\alpha} f(t)>0$ (respectively, $<0$ ), then $f$ is a strictly increasing (respectively, decreasing) function. 
Theorem 2.21. Let $f, g$ be continuous functions on $[a, b], G_{T}^{\alpha}$-differentiable on $(a, b)$ for some $\alpha \in(0,1]$. If $G_{T}^{\alpha} f(t) \geq G_{T}^{\alpha} g(t)$ for all $t \in(a, b)$, then the following statements hold:

(1) If $f(a)=g(a)$, then $f(t) \geq g(t)$ for all $t \in[a, b]$.

(2) If $f(b)=g(b)$, then $f(t) \leq g(t)$ for all $t \in[a, b]$.

Proof. Consider the function $h(t)=f(t)-g(t)$. Then $h$ is continuous on $[a, b]$ and $G_{T}^{\alpha}$-differentiable on $(a, b)$. Theorem 2.5 gives that $G_{T}^{\alpha} h(t)=G_{T}^{\alpha} f(t)-G_{T}^{\alpha} g(t) \geq 0$ for all $t \in(a, b)$, and so $h$ is a non-decreasing function by Theorem 2.20 . Hence, $h(a) \leq h(t) \leq h(b)$ for every $t \in[a, b]$.

If $h(a)=f(a)-g(a)=0$, then $f(t)-g(t)=h(t) \geq h(a)=0$ for every $t \in[a, b]$.

If $h(b)=f(b)-g(b)=0$, then $f(t)-g(t)=h(t) \leq h(b)=0$ for every $t \in$ $[a, b]$.

We can use again Rolle's theorem (Theorem 2.17) to prove the following result, which generalizes the mean value theorem (Theorem 2.18).

Theorem 2.22. Let $f, g:[a, b] \rightarrow \mathbb{R}$ be continuous functions, $G_{T}^{\alpha}$-differentiable on $(a, b)$ for some $\alpha \in(0,1]$. Then there exists $c \in(a, b)$ such that

$$
(g(b)-g(a)) G_{T}^{\alpha} f(c)=(f(b)-f(a)) G_{T}^{\alpha} g(c) .
$$

Furthermore, if $g(a) \neq g(b)$ and $G_{T}^{\alpha} g(c) \neq 0$, then

$$
\frac{G_{T}^{\alpha} f(c)}{G_{T}^{\alpha} g(c)}=\frac{f(b)-f(a)}{g(b)-g(a)} .
$$

Proof. Let us define

$$
F(t)=(g(b)-g(a))(f(t)-f(a))-(f(b)-f(a))(g(t)-g(a)) .
$$

Since $F$ satisfies the assumptions of Rolle's theorem (Theorem 2.17), there exists $c \in(a, b)$ such that $G_{T}^{\alpha} F(c)=0$. Hence,

$$
G_{T}^{\alpha} F(c)=(g(b)-g(a)) G_{T}^{\alpha} f(c)-(f(b)-f(a)) G_{T}^{\alpha} g(c)=0,
$$

and this gives the result.

This result has a direct consequence which is a fractional version of BernoulliL'Hôpital's rule.

Corollary 2.23. Let $f, g$ be functions such that $\lim _{t \rightarrow a} f(t)=\lim _{t \rightarrow a} g(t)=0$. If there exists $\lim _{t \rightarrow a} G_{T}^{\alpha} f(t) / G_{T}^{\alpha} g(t)$ for some $\alpha \in(0,1]$, then

$$
\lim _{t \rightarrow a} \frac{f(t)}{g(t)}=\lim _{t \rightarrow a} \frac{G_{T}^{\alpha} f(t)}{G_{T}^{\alpha} g(t)} .
$$

Let us state now some results on fractional derivatives of order $\alpha>1$.

Theorem 2.24. Let $I \subseteq \mathbb{R}$ be an interval, $f: I \rightarrow \mathbb{R}$ and $\alpha \in(1,2]$. If $f$ has a turning point in $t \in I$ and is $G_{T}^{\alpha}$-differentiable at $t$, then $G_{T}^{\alpha} f(t)=0$. 
Proof. We can assume that there exists $\varepsilon>0$ such that $f$ is concave in $(t, t+\varepsilon)$ and convex in $(t-\varepsilon, t)$, since otherwise we can consider $-f$ instead of $f$. Since $f$ is convex in $(t-\varepsilon, t)$, if $0<h<\varepsilon /(2 T(1, \alpha))$ then

$$
\begin{aligned}
& f(t-h T(1, \alpha)) \leq \frac{1}{2} f(t-2 h T(1, \alpha))+\frac{1}{2} f(t), \\
& \frac{f(t)-2 f(t-h T(1, \alpha))+f(t-2 h T(1, \alpha))}{h^{2}} \geq 0, \\
& G_{T}^{\alpha} f(t) \geq 0 .
\end{aligned}
$$

Since $f$ is concave in $(t, t+\varepsilon)$, a similar argument gives $G_{T}^{\alpha} f(t) \leq 0$, and we conclude that $G_{T}^{\alpha} f(t)=0$.

By using the argument in the proof of Theorem 2.24 we obtain the following result.

Theorem 2.25. Let $I \subseteq \mathbb{R}$ be an interval, $f: I \rightarrow \mathbb{R}$ a convex (respectively, concave) function and $\alpha \in(1,2]$. If $f$ is $G_{T}^{\alpha}$-differentiable at $t \in I$, then $G_{T}^{\alpha} f(t) \geq 0$ (respectively, $\left.G_{T}^{\alpha} f(t) \leq 0\right)$.

Now we present our fractional version of Flett's theorem.

Theorem 2.26. Let $f:[a, b] \rightarrow \mathbb{R}$ be a $G_{T}^{\alpha}$-differentiable function on $[a, b]$ for some $\alpha \in(0,1]$ such that $f^{\prime}(a)=f^{\prime}(b)$. Then there exists $c \in(a, b)$ such that

$$
\frac{G_{T}^{\alpha} f(c)}{T(c, \alpha)}=\frac{f(c)-f(a)}{c-a} .
$$

Proof. Without loss of generality we can assume that $f^{\prime}(a)=f^{\prime}(b)=0$, for if this is not the case, we could work with $f(t)-t f^{\prime}(a)$. Let us consider the function

$$
H(t)= \begin{cases}\frac{f(t)-f(a)}{t-a}, & t \neq a \\ 0, & t=a .\end{cases}
$$

Theorem 2.4 gives that $H$ is a continuous function on $(a, b]$; since $f^{\prime}(a)=0, H$ is continuous on $[a, b]$. Theorem 2.5 gives that $H$ is $G_{T}^{\alpha}$-differentiable on $(a, b]$. So we have, for $a<t \leq b$,

$$
G_{T}^{\alpha} H(t)=G_{T}^{\alpha}\left[\frac{f(t)-f(a)}{t-a}\right]=-\frac{(f(t)-f(a)) T(t, \alpha)}{(t-a)^{2}}+\frac{G_{T}^{\alpha} f(t)}{t-a} .
$$

If $H(b)=0$, then we have $H(a)=H(b)$ and the result is an immediate consequence of 2.1 and Rolle's theorem (Theorem 2.17).

Suppose then that $H(b) \neq 0$. We can assume that $H(b)>0$ (the case $H(b)<0$ is similar). Since $G_{T}^{\alpha} f(b)=0,2.1$ gives

$$
G_{T}^{\alpha} H(b)=-\frac{H(b) T(b, \alpha)}{b-a}<0 .
$$

By Theorem 2.20 there exists $c_{1} \in(a, b)$ such that $H\left(c_{1}\right)>H(b)$. Since $H$ is a continuous function in $\left[a, c_{1}\right]$ and $0=H(a)<H(b)<H\left(c_{1}\right)$, by the intermediate 
value theorem there is $c_{2} \in\left(a, c_{1}\right)$ such that $H\left(c_{2}\right)=H(b)$. From Rolle's theorem on $\left[c_{2}, b\right]$ we have

$$
G_{T}^{\alpha} H(c)=-\frac{(f(c)-f(a)) T(c, \alpha)}{(c-a)^{2}}+\frac{G_{T}^{\alpha} f(c)}{c-a}=0 .
$$

Corollary 2.27. Let $f, g:[a, b] \rightarrow \mathbb{R}$ be $G_{T}^{\alpha}$-differentiable functions on $[a, b]$ for some $\alpha \in(0,1]$, with $G_{T}^{\alpha} g(a) \neq 0, g(t) \neq g(a)$ for all $t \in(a, b], \frac{f^{\prime}(a)}{g^{\prime}(a)}=\frac{f^{\prime}(b)}{g^{\prime}(b)}$ and $G_{T}^{\alpha} g(b)(g(b)-b(a))>0$. Then there exists $c \in(a, b)$ such that

$$
(g(c)-g(a)) G_{T}^{\alpha} f(c)=(f(c)-f(a)) G_{T}^{\alpha} g(c) .
$$

Let $I \subseteq \mathbb{R}$ be an interval, $a, t \in I$ and $\alpha \in \mathbb{R}$. In [15] the integral operator $J_{T, a}^{\alpha}$ is defined for every locally integrable function $f$ on $I$ as

$$
J_{T, a}^{\alpha}(f)(t)=\int_{a}^{t} \frac{f(s)}{T(s, \alpha)} d s .
$$

The following result appears in [15]. We include its proof for the sake of completeness.

Proposition 2.28. Let $I \subseteq \mathbb{R}$ be an interval, $a \in I, 0<\alpha \leq 1$ and $f$ a differentiable function on $I$ such that $f^{\prime}$ is a locally integrable function on $I$. Then, we have for all $t \in I$

$$
J_{T, a}^{\alpha}\left(G_{T}^{\alpha}(f)\right)(t)=f(t)-f(a) .
$$

Proof. Since $f^{\prime}$ is a locally integrable function on $I$, Theorem 2.4 gives

$$
J_{T, a}^{\alpha}\left(G_{T}^{\alpha}(f)\right)(t)=\int_{a}^{t} \frac{G_{T}^{\alpha}(f)(s)}{T(s, \alpha)} d s=\int_{a}^{t} f^{\prime}(s) d s=f(t)-f(a),
$$

which is the desired result.

The integral operator $J_{T, a}^{\alpha}$ for the choice of the kernel $T$ given by $T(t, \alpha)=t^{1-\alpha}$ is defined in [22], and [22, Theorem 3.1] shows that

$$
G^{\alpha} J_{t^{1-\alpha}, a}^{\alpha}(f)(t)=f(t),
$$

for every continuous function $f$ on $I, a, t \in I$ and $\alpha \in(0,1]$. Hence, Proposition 2.29 (see [15]) extends to any $T$ this important equality.

Proposition 2.29. Let $I \subseteq \mathbb{R}$ be an interval, $a \in I$ and $\alpha \in(0,1]$. Then

$$
G_{T}^{\alpha}\left(J_{T, a}^{\alpha}(f)\right)(t)=f(t),
$$

for every continuous function $f$ on $I$ and $a, t \in I$.

For more information about the integral operator and its applications, see [4, 12 , 15]. The following result summarizes some elementary properties of the integral operator $J_{T, a}^{\alpha}$.

Theorem 2.30. Let $I \subseteq \mathbb{R}$ be an interval, $a, b \in I$ and $\alpha \in \mathbb{R}$. Suppose that $f, g$ are locally integrable functions on $I$, and $k_{1}, k_{2} \in \mathbb{R}$. Then we have

(1) $J_{T, a}^{\alpha}\left(k_{1} f+k_{2} g\right)(t)=k_{1} J_{T, a}^{\alpha} f(t)+k_{2} J_{T, a}^{\alpha} g(t)$; 
(2) if $f \geq g$, then $J_{T, a}^{\alpha} f(t) \geq J_{T, a}^{\alpha} g(t)$ for every $t \in I$ with $t \geq a$;

(3) $\left|J_{T, a}^{\alpha} f(t)\right| \leq J_{T, a}^{\alpha}|f|(t)$ for every $t \in I$ with $t \geq a$;

(4) $\int_{a}^{b} \frac{f(s)}{T(s, \alpha)} d s=J_{T, a}^{\alpha} f(t)-J_{T, b}^{\alpha} f(t)=J_{T, a}^{\alpha} f(t)(b)$ for every $t \in I$.

\section{ACKNOWLEDGEMENT}

We would like to thank the referees for their work, which has improved the presentation of the paper.

\section{REFERENCES}

[1] T. Abdeljawad, On conformable fractional calculus, J. Comput. Appl. Math. 279 (2015), 57-66. MR 3293309.

[2] M. Al Horani and R. Khalil, Total fractional differentials with applications to exact fractional differential equations, Int. J. Comput. Math. 95 (2018), no. 6-7, 1444-1452. MR 3783192

[3] R. Almeida, M. Guzowska and T. Odzijewicz, A remark on local fractional calculus and ordinary derivatives, Open Math. 14 (2016), no. 1, 1122-1124. MR 3592616

[4] A. Atangana, D. Baleanu and A. Alsaedi, New properties of conformable derivative, Open Math. 13 (2015), no. 1, 889-898. MR 3430934

[5] A. Atangana and E. F. Doungmo Goufo, Extension of matched asymptotic method to fractional boundary layers problems, Math. Probl. Eng. 2014, Art. ID 107535, 7 pp. MR 3283450

[6] A. Atangana and D. Baleanu, New fractional derivatives with nonlocal and non-singular kernel: Theory and application to heat transfer model, Therm Sci. 20 (2016), no. 2, 763 769.

[7] D. Baleanu, A. Jajarmi and J. H. Asad, Classical and fractional aspects of two coupled pendulums, Rom. Rep. Phys. 71 (2019), Art. no. 103.

[8] D. Baleanu, S. Sadat Sajjadi, A. Jajarmi and J. H. Asad, New features of the fractional EulerLagrange equations for a physical system within non-singular derivative operator, Eur. Phys. J. Plus 134 (2019), Art. no. 181.

[9] D. Baleanu, J. H. Asad and A. Jajarmi, The fractional model of spring pendulum: new features within different kernels, Proc. Rom. Acad. Ser. A Math. Phys. Tech. Sci. Inf. Sci. 19 (2018), no. 3, 447-454. MR 3847759

[10] D. Baleanu, J. H. Asad and A. Jajarmi, New aspects of the motion of a particle in a circular cavity, Proc. Rom. Acad. Ser. A Math. Phys. Tech. Sci. Inf. Sci. 19 (2018), no. 2, 361-367. MR 3816718

[11] R. A. Blaya, R. Ávila and J. B. Reyes, Boundary value problems with higher order Lipschitz boundary data for polymonogenic functions in fractal domains, Appl. Math. Comput. 269 (2015), 802-808. MR 3396822

[12] P. Bosch, J. F. Gómez-Aguilar, J. M. Rodríguez and J. M. Sigarreta, Analysis of dengue fever outbreak by generalized fractional derivative, Fractals 28 (2020), no. 8, Art. 2040038.

[13] K. Diethelm et al., Why fractional derivatives with nonsingular kernels should not be used, Fract. Calc. Appl. Anal. 23 (2020), no. 3, 610-634. MR 4124293.

[14] A. Fernandez, M. A. Özarslan and D. Baleanu, On fractional calculus with general analytic kernels, Appl. Math. Comput. 354 (2019), 248-265. MR 3921011.

[15] A. Fleitas, J. F. Gómez-Aguilar, J. E. Nápoles Valdés, J. M. Rodríguez and J. M. Sigarreta, Analysis of the local Drude model involving the generalized fractional derivative, Optik 193 (2019), 163008 . 
[16] J. F. Gómez-Aguilar, Irving-Mullineux oscillator via fractional derivatives with Mittag-Leffler kernel, Chaos Solitons Fractals 95 (2017), 179-186. MR 3597510.

[17] P. M. Guzmán et al., A new definition of a fractional derivative of local type, J. Math. Anal. 9 (2018), no. 2, 88-98. MR 3797479

[18] M. Hajipour et al., On an accurate discretization of a variable-order fractional reaction-diffusion equation, Commun. Nonlinear Sci. Numer. Simul. 69 (2019), 119-133. MR 3887058

[19] M. Ilie, J. Biazar and Z. Ayati, The first integral method for solving some conformable fractional differential equations, Opt. Quantum Electron. 50 (2018), no. 2, Article no. 55.

[20] F. Jarad et al., On a new class of fractional operators, Adv. Difference Equ. 2017, Paper No. 247, 16 pp. MR 3688989

[21] V. N. Katugampola, A new fractional derivative with classical properties. https://arxiv. org/abs/1410.6535 [math.CA], 2014.

[22] R. Khalil et al., A new definition of fractional derivative, J. Comput. Appl. Math. 264 (2014), 65-70. MR 3164103.

[23] A. A. Kilbas, H. M. Srivastava and J. J. Trujillo, Theory and applications of fractional differential equations, North-Holland Mathematics Studies, 204, Elsevier Science, Amsterdam, 2006. MR 2218073

[24] K. S. Miller, Fractional differential equations, J. Fract. Calc. 3 (1993), 49-57. MR 1218092.

[25] F. Mohammadi et al., A hybrid functions numerical scheme for fractional optimal control problems: application to nonanalytic dynamic systems, J. Vib. Control 24 (2018), no. 21, 5030-5043. MR 3869396.

[26] K. B. Oldham and J. Spanier, The fractional calculus, Mathematics in Science and Engineering, Vol. 111, Academic Press, New York, 1974. MR 0361633

[27] B. Shiri and D. Baleanu, System of fractional differential algebraic equations with applications, Chaos Solitons Fractals 120 (2019), 203-212. MR 3910534.

\section{A. Fleitas}

Universidad Autónoma de Guerrero, Centro Acapulco, CP 39610, Acapulco de Juárez, Guerrero, México

satielfo@gmail.com

\section{J. E. Nápoles Valdés}

Universidad Nacional del Nordeste, Facultad de Ciencias Exactas y Naturales y Agrimensura, 3400 Corrientes, Argentina

jnapoles@exa.unne.edu.ar

\section{J. M. Rodríguez}

Universidad Carlos III de Madrid, Departamento de Matemáticas, Avenida de la Universidad 30, 28911 Leganés, Madrid, Spain

jomaro@math.uc3m.es

J. M. Sigarreta-Almira ${ }^{\bowtie}$

Facultad de Matemáticas, Universidad Autónoma de Guerrero, Guerrero, México

josemariasigarretaalmira@hotmail.com

Received: February 25, 2020

Accepted: July 28, 2020 